\title{
DERECHOS PRIORITARIOS DE AGUA: ¿SON UNA OPCIÓN ADECUADA PARA LA ASIGNACION DE AGUA DURANTE PERIODOS DE SEQUÍA?
}

\author{
José A. Gómez-Limón, Carlos Gutiérrez Martín* y Nazaret M. Montilla-López \\ WEARE-Water, Environmental and Agricultural Resources Economics Research Group, University of \\ Cordoba (Cordoba, jglimon@uco.es, carlos.gutierrez@uco.es, g02molon@uco.es)
}

\section{Resumen}

Este trabajo examina la conveniencia de reformar la asignación de agua en el sector del regadío, reemplazando el actual sistema de asignación basado en la regla proporcional por la implementación de una regla de prioridad que establezca derechos de agua diferenciados por su grado de garantía de suministro. El objetivo principal consiste en evaluar si este cambio en los derechos de agua puede considerarse una alternativa razonable a los mercados de agua con el fin de mejorar la eficiencia económica de la asignación del agua durante situaciones de escasez coyuntural a nivel de comunidad de regantes. Para ello, se construye un modelo de programación matemática para poder simular el desempeño de la reforma propuesta en una comunidad de regantes del sur de España. Los resultados muestran que las ganancias de eficiencia provocadas por este cambio en el sistema de asignación son muy pequeñas. Por tanto, este instrumento de gestión de la demanda no puede considerarse como un sustitutivo adecuado de los mercados de agua.

Palabras clave: Asignación de agua, Escasez coyuntural, Derechos prioritarios de agua, Eficiencia, Programación matemática, Guadalquivir.

\section{Introducción}

En el sector del regadío, la regla proporcional es el método de racionamiento utilizado para la asignación del agua de riego durante las sequías hidrológicas. Según esta regla, todos los titulares de concesiones de agua reciben una cantidad de agua proporcional a sus derechos, de modo que la cantidad total del recurso usada se iguale a las disponibilidades. Existen otros sistemas de racionamiento de agua, como el de la regla de prioridad, mediante el cual los titulares de los derechos de riego se dividen en clases de prioridad, siendo sus derechos de agua asignados lexicográficamente según los niveles de prioridad establecidos. Esto significa que las demandas de los titulares de derechos con mayor prioridad se atienden en primer lugar, y solo cuando están plenamente satisfechas, se asignan los recursos restantes a los siguientes titulares de derechos según un criterio de orden de prioridad decreciente [Gómez-Limón et al. (2020)].

El objetivo de este trabajo es evaluar la mejora en la eficiencia económica que potencialmente podría generarse si se reemplaza el actual régimen de asignación basado en la regla proporcional por un régimen basado en una regla de asignación por prioridades a escala de comunidad de regantes (CR). Con ese fin, nos centramos en la CR del Sector BXII del Bajo Guadalquivir (SBXII), en el sur de España, como caso de estudio. La evaluación empírica del cambio propuesto se realiza utilizando un modelo de programación matemática que simula las decisiones de los regantes, entre las que destacan las relativas a la composición de sus carteras individuales de derechos de agua, integrando derechos 'prioritarios' y derechos 'generales'.

\section{Caso de estudio}

La CR del SBXII es un distrito de riego modernizado de 14.643 hectáreas, repartidas entre 569 explotaciones. En zona regable se identificó una tipología de explotaciones a través del análisis de conglomerados, utilizando el porcentaje de área dedicada a cada cultivo como variables diferenciadoras [para más detalles, véase Montilla-López et al. (2018)]. La explotación tipo 1 (ET1) es representativa del $38 \%$ de las explotaciones y del $52 \%$ de la superficie regada de la CR, orientada mayoritariamente a hortícolas, algodón y remolacha. La explotación tipo 2 (ET2) representa el $41 \%$ de las explotaciones y el $36 \%$ del total de superficie, y es la que más diversifica los cultivos. Por último, la explotación tipo 3 (ET3) representa el $20 \%$ de las explotaciones y el $11 \%$ de la superficie total de la CR, y está dedicada a los cultivos más extensivos, como algodón y remolacha. Estas explotaciones tipo son las unidades de análisis consideradas en el modelo de programación construido para simular el desempeño del instrumento propuesto.

La concesión de agua asciende a $6.000 \mathrm{~m}^{3} /$ ha, que solo puede dotarse en años hidrológicos 'normales', cuando la disponibilidad de agua para riego es suficiente para atender todas las demandas. La estimación de la distribución de probabilidad de las asignaciones anuales de agua a la CR Sector BXII se basa en un modelo de simulación hidrológica estocástica considerando la situación actual de oferta y demanda de agua en la cuenca del Guadalquivir, así como el marco institucional fijado en los planes hidrológicos de la cuenca y su plan de gestión de sequía [CHG (2015)]. Este modelo ha sido desarrollado por Gómez-Limón (2020) 
para el sistema hidrológico donde se ubica el caso de estudio (Sistema de Regulación General), obteniendo el histograma de dotaciones anuales de agua que se muestra en el Gráfico 1. La función de distribución resultante no se ajusta a ninguna función de uso común. Por este motivo su empleo en el ejercicio de programación planteado se ha hecho simulando observaciones de la misma de manera aleatoria.

Gráfico 1. Función distribución de la dotación anual de agua de riego en el Sistema de Regulación General de la Cuenca del Guadalquivir

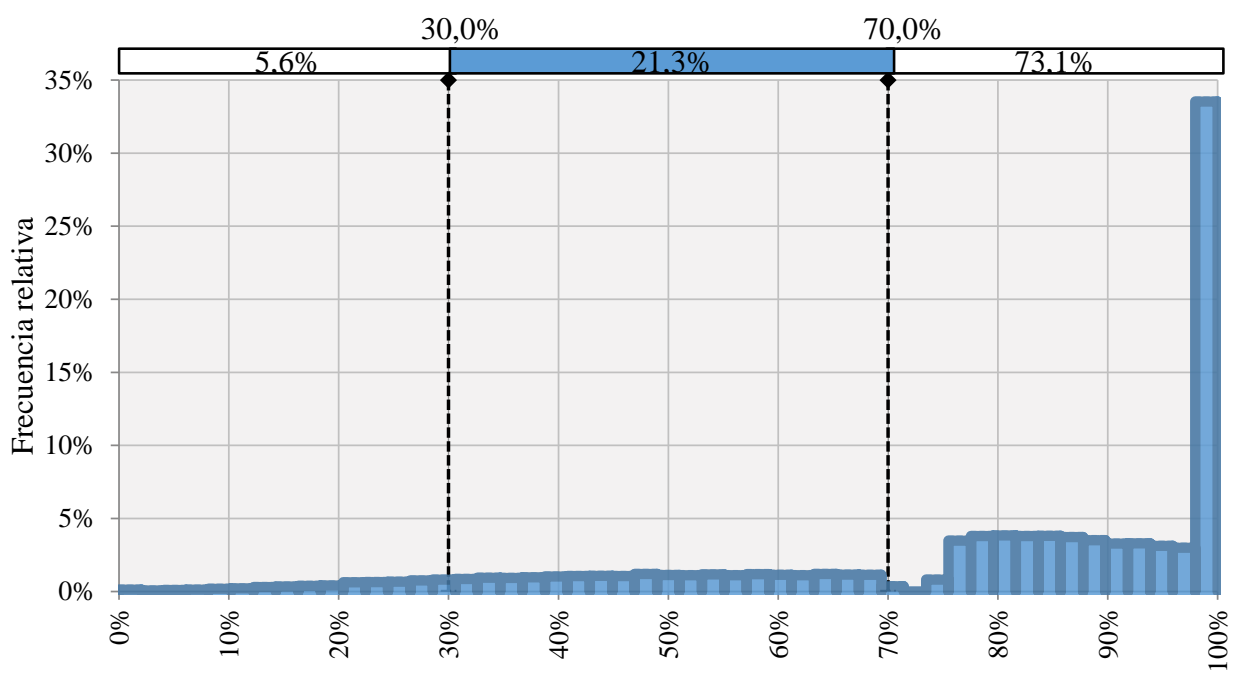

Dotación anual de agua de riego / Derecho concesional (maxima dotación)

Fuente: Gómez-Limón (2020).

\section{Simulación mediante programación matemática}

Siguiendo el ejemplo australiano, se proponen dos clases de prioridad, distinguiendo entre derechos de alta seguridad o 'prioritarios' y derechos de agua de baja seguridad o 'generales'. Los regantes podrían combinar estas dos clases creando una cartera de derechos de agua para lograr cualquier nivel deseado de garantía de suministro. Por tanto, la asignación de agua por hectárea a cada explotación tipo será una combinación del agua asignada a cada uno de sus derechos, teniendo en cuenta el porcentaje proveniente de derechos prioritarios y generales. Solo una parte de los derechos totales de agua de la CR serían elegibles para actualizarse a derechos prioritarios. Los derechos prioritarios se asignarían a aquellos regantes dispuestos a asumir un sobrecargo en la derrama de riego, dispuesta para compensar a los titulares derechos generales por la pérdida de garantía de suministro que este nuevo sistema de asignación les generaría.

La asignación de agua a cada derecho prioritario y general (y por ende a cada explotación tipo) se determina anualmente en función del agua asignada a la CR por el organismo de cuenca, siguiendo el procedimiento de prioridad antes mencionado. Por lo tanto, los derechos prioritarios se atienden primero con la asignación de agua completa otorgada a la CR, siempre que la disponibilidad de agua sea suficiente para cubrir todas las demandas de agua de los titulares de derechos prioritarios. Después de que dicha demanda quede cubierta, el agua restante se distribuye proporcionalmente entre los titulares de derechos generales. En el caso de no disponer de agua suficiente para cumplir con las dotaciones completas de derechos prioritarios, el agua disponible se racionará entre los titulares de derechos prioritarios siguiendo la regla proporcional. En este último caso, los titulares de derechos generales no recibirían ninguna dotación de agua.

La toma de decisión de los titulares de cada explotación tipo se simula a través de un modelo basado en la Programación Matemática Positiva. En concreto, la técnica seguida es la planteada originalmente por Howitt (1995), con una función de costes cuadrática específica para cada cultivo, considerando la aproximación del coste medio propuesta por Heckelei y Britz (2005). En este contexto, se asumen que los agricultores buscan maximizar el promedio de su margen bruto para el conjunto de los escenarios de simulación planteados $(N=5000$ valores probabilísticos de la dotación de agua para riego a la CR extraídos de la distribución arriba señalada), considerando como variables de decisión la superficie que se asigna a cada cultivo y el porcentaje de derechos prioritarios de su cartera de derechos, teniendo como restricciones la limitación de superficie, agua disponible, rotaciones de cultivos y restricciones de la PAC para el algodón y remolacha. Para encontrar el porcentaje óptimo de derechos prioritarios a nivel de CR, dicho porcentaje se introduce en el modelo como una restricción adicional y se parametriza su valor con incrementos de 
0,5\%. La solución óptima será aquella en la que se obtenga una mayor eficiencia económica, medida como la suma de los márgenes brutos del conjunto de explotaciones tipo analizadas.

\section{Resultados y discusión}

Los resultados del modelo de simulación arrojan la evidencia de que los derechos prioritarios podrían mejorar la eficiencia económica a nivel de CR. De hecho, en comparación con los valores de referencia del régimen actual de derechos de agua proporcionales, el margen bruto promedio a nivel de CR aumenta para todos los valores del porcentaje máximo de derechos prioritarios considerados en la parametrización realizada. Sin embargo, las mejoras de la eficiencia económica estimadas son casi insignificantes, registrando un máximo del $0,2 \%$ del margen bruto total de la $\mathrm{CR}$ en el escenario actual de asignación proporcional. Esta máxima mejora de eficiencia se obtiene para un valor óptimo de derechos prioritarios del 20,5\% del total de los derechos de agua asignados a la CR.

La Tabla 1 muestra los resultados de la simulación para la solución de máxima eficiencia, basados en una participación del 20,5\% de los derechos de agua prioritarios otorgados a nivel de CR. Tomando esta solución como el mejor resultado posible, se puede observar que la ET1 optimizaría su beneficio poseyendo en su cartera de derechos de agua el 30,7\% de derechos a prioritarios. Sin embargo, cabe señalar que ET2 y ET3 también mejoran una parte de sus derechos de agua, aunque en menores proporciones $(11,3 \%$ y $3,1 \%$, respectivamente). Esta asignación óptima de derechos de agua prioritarios permite a ET1 aumentar su asignación de agua promedio anual en comparación con la situación actual (implementación de derechos de agua proporcionales), consiguiendo además que esta asignación de agua se vuelva más estable con el tiempo (un aumento del 3,2\% en la asignación promedio y una disminución del 11,3\% en su desviación estándar). En consecuencia, esta garantía mejorada del suministro de agua conduce a un aumento en el margen bruto promedio (2,0\%) y una disminución en su variabilidad (reducción del 18,0\% en su desviación estándar).

Por otro lado, la garantía de suministro de agua de riego empeora para ET2 y ET3, tanto en términos de la media (descensos del 2,9\% y 5,5\%, respectivamente) como de su variabilidad (incrementos del 10,3\% y $19,5 \%$, respectivamente, en sus desviaciones estándar). Este deterioro de la garantía de suministro conduce a peores indicadores de rentabilidad: reducción de los márgenes brutos promedio $(2,1 \% \mathrm{y} 5,0 \%$, respectivamente) y aumento de la volatilidad del margen bruto (aumento del 22,1\% en la desviación estándar del margen bruto para ambas explotaciones tipo). Sin embargo, estas pérdidas teóricamente podrían compensarse en su totalidad con los recargos sobre las derramas de la CR pagados por los titulares de la ET1.

Tabla 1. Resultados del modelo de simulación para la solución de máxima eficiencia

\begin{tabular}{|c|c|c|c|c|c|}
\hline & & ET1 & ET2 & ET3 & $\mathbf{C R}$ \\
\hline \multicolumn{2}{|c|}{ Derechos de agua con prioridad (\%) } & $30,7 \%$ & $11,3 \%$ & $3,1 \%$ & $20,5 \%$ \\
\hline \multirow{3}{*}{$\begin{array}{l}\text { Asignación de agua } \\
\text { anual media } \\
\left(\mathrm{m}^{3} / \mathrm{ha}\right)\end{array}$} & Regla proporcional & $4.753,2$ & $4.753,2$ & $4.753,2$ & $4.753,2$ \\
\hline & Regla prioritaria & $4.906,1$ & $4.615,7$ & $4.492,8$ & $4.753,2$ \\
\hline & $\begin{array}{l}\text { Incremento (Prioritaria- } \\
\text { Proporcional) }\end{array}$ & $\begin{array}{l}152,9 \\
(3,2 \%)\end{array}$ & $\begin{array}{l}-137,5 \\
(-2,9 \%)\end{array}$ & $\begin{array}{l}-260,4 \\
(-5,5 \%)\end{array}$ & $\begin{array}{c}0,0 \\
(0,0 \%)\end{array}$ \\
\hline \multirow{3}{*}{$\begin{array}{c}\text { Desviación estándar } \\
\text { de la asignación de } \\
\text { agua anual } \\
\left(\mathrm{m}^{3} / \mathrm{ha}\right)\end{array}$} & Regla proporcional & $1.454,6$ & $1.454,6$ & $1.454,6$ & $1.454,6$ \\
\hline & Regla prioritaria & $1.290,0$ & $1.604,2$ & $1.738,9$ & $1.454,6$ \\
\hline & $\begin{array}{l}\text { Incremento (Prioritaria- } \\
\text { Proporcional) }\end{array}$ & $\begin{array}{c}-164,6 \\
(-11,3 \%)\end{array}$ & $\begin{array}{c}149,6 \\
(10,3 \%)\end{array}$ & $\begin{array}{c}284,3 \\
(19,5 \%)\end{array}$ & $\begin{array}{c}0,0 \\
(0,0 \%)\end{array}$ \\
\hline \multirow{3}{*}{$\begin{array}{l}\text { Margen bruto anual } \\
\text { medio } \\
(€ / \text { ha })\end{array}$} & Regla proporcional & $2.640,1$ & $1.990,8$ & $1.586,5$ & $2.283,5$ \\
\hline & Regla prioritaria & $2.693,2$ & $1.949,3$ & $1.507,7$ & $2.287,1$ \\
\hline & $\begin{array}{l}\text { Incremento (Prioritaria- } \\
\text { Proporcional) }\end{array}$ & $\begin{array}{c}53,2 \\
(2,0 \%)\end{array}$ & $\begin{array}{c}-41,5 \\
(-2,1 \%)\end{array}$ & $\begin{array}{c}-78,8 \\
(-5,0 \%)\end{array}$ & $\begin{array}{c}3,6 \\
(0,2 \%)\end{array}$ \\
\hline \multirow{3}{*}{$\begin{array}{c}\text { Desviación estándar } \\
\text { del margen bruto anual } \\
(€ / \mathrm{ha})\end{array}$} & Regla proporcional & 429,9 & 315,4 & 334,2 & 375,8 \\
\hline & Regla prioritaria & 352,6 & 385,3 & 445,6 & 372,4 \\
\hline & $\begin{array}{l}\text { Incremento (Prioritaria- } \\
\text { Proporcional) }\end{array}$ & $\begin{array}{c}-77,3 \\
(-18,0 \%)\end{array}$ & $\begin{array}{c}69,8 \\
(22,1 \%)\end{array}$ & $\begin{array}{c}111,4 \\
(22,1 \%)\end{array}$ & $\begin{array}{c}-3,4 \\
(-0,9 \%)\end{array}$ \\
\hline
\end{tabular}


Es importante destacar que el volumen de agua total asignado a nivel de CR es el mismo que en el escenario de referencia con reparto proporcional, por lo que el cambio en el sistema de reparto propuesto no tiene impactos sobre el medioambiente.

Si nos centramos en los resultados específicos para distintas disponibilidades de agua en vez del resultado promedio de las dotaciones de agua probabilísticas $(N=5000)$, se observa cómo las mayores mejoras de eficiencia respecto al escenario base de reparto proporcional se producen para los niveles de sequía más extremos (dotaciones a la CR del 10-20\% de los derechos concesionales). Para sequías moderadas (dotaciones a la CR del 70-90\% de los derechos concesionales), las mejoras de eficiencia son menores. Finalmente, se observa que para sequías severas (40-60\% de la disponibilidad total), el escenario de referencia con la regla de reparto proporcional es más eficiente que el reparto por derechos con derechos prioritarios de suministro.

En cualquier caso, debe aclararse que los resultados de la simulación realizada se corresponden a una implementación idealizada del instrumento propuesto, considerando costes de transacción nulos. Por este este motivo, teniendo las exiguas mejoras de eficiencias que se prevé alcanzar en este escenario ideal, es razonable pensar que la implementación de los derechos prioritarios en el mundo real no generaría incrementos de eficiencias a nivel de CR, por lo que debe descartarse como instrumento de gestión de la demanda sustitutivo de los mercados de agua.

\section{Conclusiones}

Aunque el desempeño simulado muestra que la reforma planteada en el sistema de asignación del agua a nivel de CR podría generar una leve mejora en la eficiencia, las ganancias económicas en el mundo real no serían suficientes para cubrir los costes de transacción asociados con la implementación del régimen de asignación prioritario propuesto. Por lo tanto, cabe concluir afirmando que los derechos de prioridad no pueden considerarse un régimen de racionamiento de agua razonable para reducir la ineficiencia de la asignación de agua causada por la asignación proporcional de agua durante los eventos cíclicos de escasez. En cualquier caso, esta conclusión resulta cierta solo para el análisis de la gestión interna del agua dentro de una comunidad de regantes, donde el valor marginal del agua no difiere demasiado de una explotación tipo a otra. La evaluación de impactos de la implementación de los derechos prioritarios a nivel de cuenca, incorporando más heterogeneidad de explotaciones (valor marginal del agua), podría proporcionar nuevos hallazgos relevantes sobre este tema.

\section{Referencias}

CHG (Confederación Hidrográfica del Guadalquivir). (2015). Plan Hidrológico de la Demarcación del Guadalquivir (2015-2021). Confederación Hidrográfica del Guadalquivir, Sevilla, Spain.

Gómez-Limón, J.A. (2020). "Hydrological drought insurance for irrigated agriculture in southern Spain". Agricultural Water Management, 240: 106271.

Gómez-Limón, J.A., Gutiérrez-Martín, C. y Montilla-López, N.M. (2020). “Agricultural water allocation under cyclical scarcity: the role of priority water rights". Water, 12(6): 1835.

Heckelei, T. y Britz, W. (2005). "Models based on Positive Mathematical Programming: state of the art and further extensions". En Arfini, F. (Ed.), Modelling Agricultural Policies: State of the Art and New Challenges. University of Parma, Parma, Italy: 48-73.

Howitt, R.E. (1995). "Positive Mathematical Programming”. American Journal of Agricultural Economics, 77(2): 329-342.

Montilla-López, N.M., Gómez-Limón, J.A. y Gutiérrez-Martín, C. (2018). “Sharing a river: potential performance of a water bank for reallocating irrigation water". Agricultural Water Management, 200: 47-59. 